\title{
Integrated Approach to Nature as Source of New Drug Lead
}

\section{Seema Kohli}

Additional information is available at the end of the chapter

http://dx.doi.org/10.5772/intechopen.74961

\begin{abstract}
Classically, the development and launching of a new drug is a highly time consuming, tedious and expensive process involving following fundamental steps: (1) Identification of cause of Disease and Search for target site. (2) Search and Optimisation of active compound, that is, the Drug Lead. (3) Testing of Drug in Animals (pre-clinical phase). (4) Clinical Trials. (5) Approval of New Drug by Competent authority and availability of the drug. Drug discovery and development process involves around 10-15 years of investigation period and incredibly high cost and investment. This process also involves participation of experts from various disciplines and fields. Therefore, the new approaches are obligatory to be developed not only to expedite the process but also to ensure the launch of safer and effective drug. Over this background, the importance of experimental wisdom and holistic approach is intensifying to offer good base as an attractive discovery engine. Natural product drug discovery, ethno-pharmacology, traditional and attractive medicines are re-emerging as new strategic options. In the past decade, the number of new chemical entity (NCG) in drug development channel is declining markedly might have led to the rekindling of interest in emergence of natural product as new drug leads. The novel natural products can be optimised on the basis of their biological activities using highly sophisticated combinatorial biosynthetic techniques, microbial genomes and screening process.
\end{abstract}

Keywords: natural products, drug leads, microorganism and marine source

\section{Introduction}

Drug discovery and development is mainly concerned with new chemical entity with biological activity. It works on enhancing the properties of drugs used in the treatment of different 
medical conditions. Classically, the development and launching of a new drug is a highly timeconsuming, tedious and expensive process involving under mentioned fundamental steps:

- Identification of cause of Disease and Search for target site

- Search and Optimisation of active compound, that is, the Drug Lead

- Testing of Drug in Animals (pre-clinical phase)

- Clinical Trials

- Approval of New Drug by Competent authority and availability in market.

Drug discovery leading to strong and doable lead candidate always remained exigent assignment for scientists. In fact experts accomplish the task by transforming the screening hit compound to a suitable drug candidate. The journey of new drug to the market is considerably long and takes about 10-15 years of investigation period. Therefore, the new approaches are obligatory to be developed not only to expedite the process but also to ensure the launch of safer and effective drug [1].

Over this background, the importance of experimental wisdom and holistic approach is intensifying to offer good base as an attractive discovery engine. Natural product drug discovery, ethno-pharmacology, traditional and attractive medicines are re-emerging as new strategic options. In the past decade, the number of new chemical entity (NCE) in drug development channel is declining markedly might have led to the rekindling of interest in the emergence of natural product new drug leads. The novel-natural products can be optimised on the basis of their biological activities using highly sophisticated combinatorial biosynthetic techniques, microbial genomes and screening process (Figure 1).

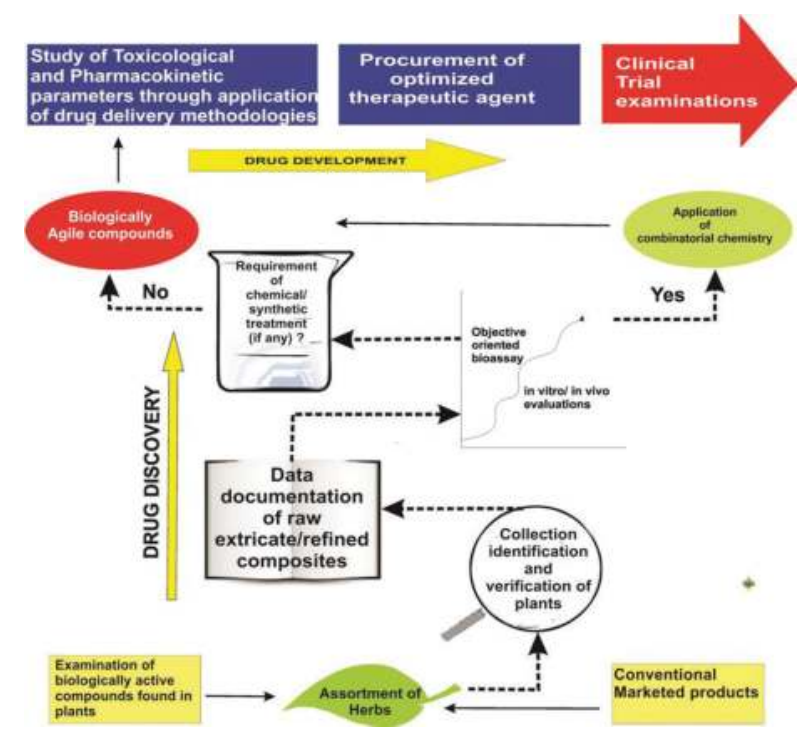

Figure 1. Complete process of drug discovery from plants. 
Since ages the natural products have been the source of medicinal agents and will continue to play crucial role in the human health through the expanded investigation of world biodiversity. World Health Organization (WHO) reports that about $80 \%$ of the world's population depends on traditional medicine for their health care. Further, at least 119 important chemical substances have been derived from 90 plant species [2].

With the advent of theory of drug-receptor action, the scientists concluded that it is the isolated compound from the plant extract that is responsible for pharmacological action. This leads to new era in pharmacology and area of new drug research. The classical example is morphine (from opium) and digoxin (from Digitalis purpurea). A number of modern medicines have been obtained from natural resources such as plants, microorganisms, marine organisms and minerals. Nature continues to be a main source of molecular diversity, which through the pursuit of multidisciplinary, international collaborative research can result in the development of promising lead compounds [3].

\section{Natural product as new drug lead}

Lead identification/optimisation is the one of the most important steps in drug development following the biological target identification. The properties of a drug can be enhanced or potentiated by making certain modifications/alterations in its chemical structure. Drug efficacy, potency, selectivity and pharmacokinetic parameters can be improved by making necessary structural changes. The chemical structure is the key to lead compound identification. After the lead compound identification, the next step is the study of ADMET that is absorption, distribution, metabolism, excertion and toxicology of the probable drug lead. If these studies are positive and satisfactory, the compound is nontoxic and nonmutagenic, then the compound is turned to be potential lead compound. This may then be developed as new drug. (Figure 2). Lead compound is a chemical compound that shows desired pharmacological activity and may initiate the development of new chemical entity, relevant compound. These are actually the starting molecule for the new drug. Newer techniques can be adopted to accelerate the enhancement in the compounds pharmacological properties.

The promising sources of lead compound and novel drugs are:

- Natural products

- Chemical libraries

- Computational Medicinal Chemistry

Recently, there has been a keen interest in natural product research as the traditional method of drug discovery failed to yield desired lead compound particularly in areas such as immunosuppressant, anti-infective and metabolic diseases. Natural product research continues to explore a variety of lead chemical structures that can be used as a template for new drug by the pharmaceutical industry. This is also evident that new approaches to enhance the joint drug discovery and development process would be expected to take place basically from 


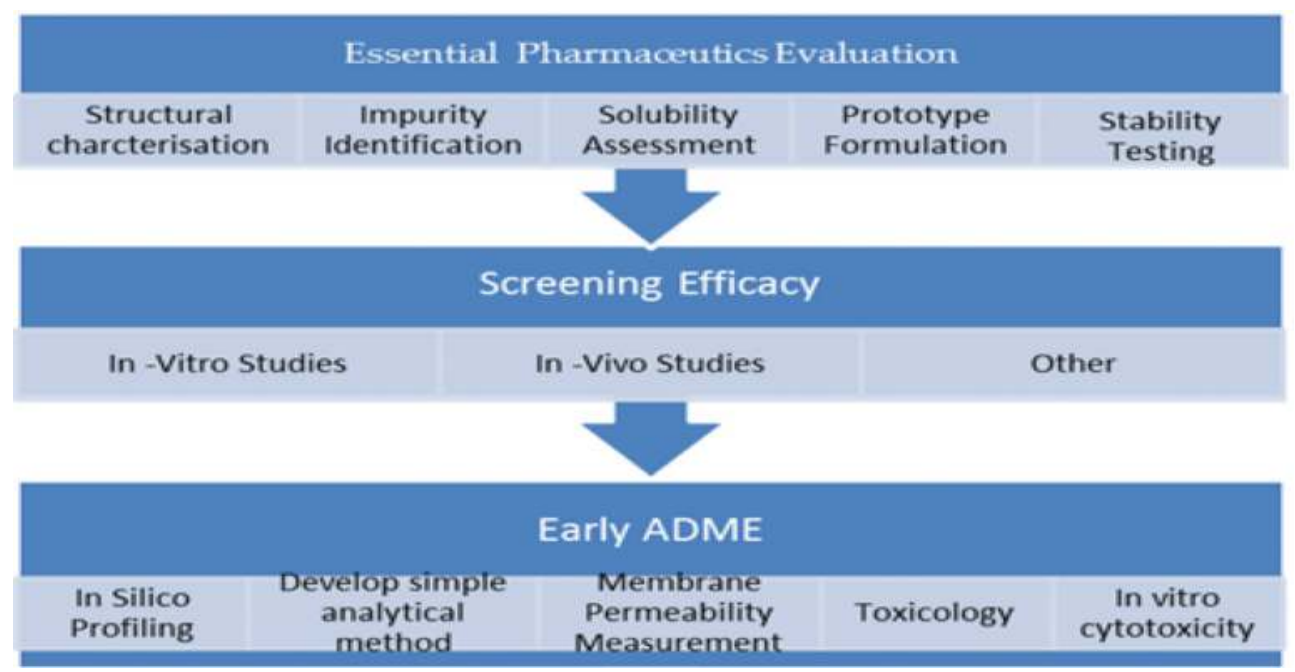

Figure 2. Process of lead selections and identification.

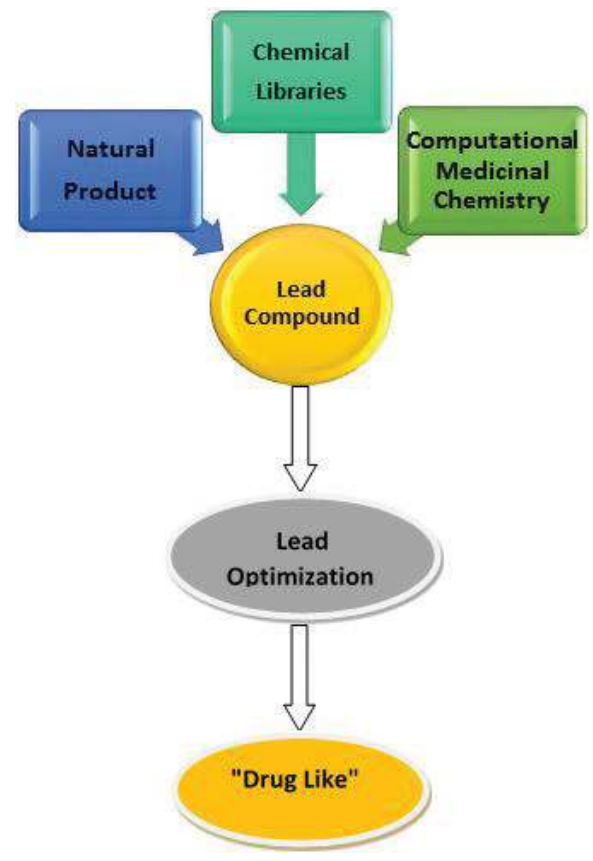

Figure 3. Drug leads and drug development.

innovation in drug target elucidation along with lead structure discovery. There are new technologies like automated separation techniques, high throughput screening and combinatorial chemistry are powerful and revolutionising drug discovery. (Figure 3). 


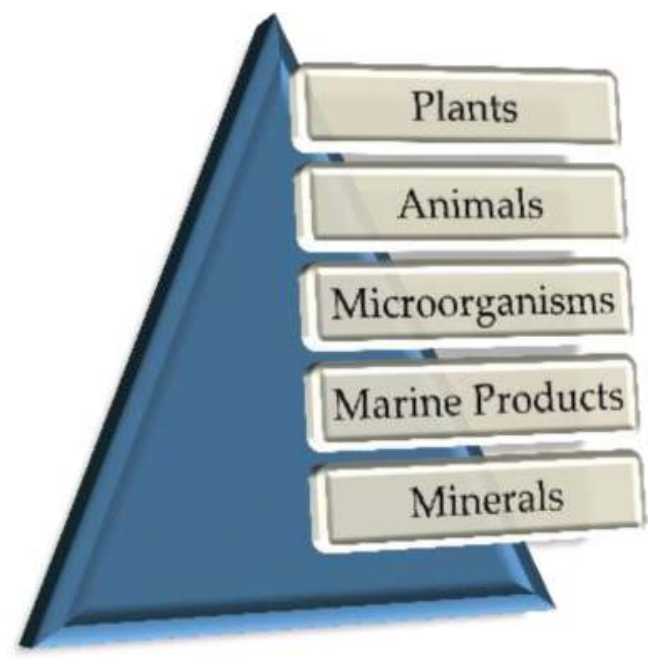

Figure 4. Natural products as drug leads.

Apart from comparing with other drug discovery methods, natural products are still providing their fair share of new clinical candidates and drugs. These said compounds were still a significant source of new drugs, especially in the anticancer, anti-infective, antihypertensive, immune-suppression and neurological disease therapeutic areas [4-7]. The natural products used as drug lead are shown in Figure 4.

\subsection{Plants}

Plants are affluent source of pharmaceuticals as well as drug leads. They are the natural laboratories where the simple chemical skeleton is transformed to complex chemical structures. The natural metabolites are far better than the synthesised metabolites in biological efficacy. A survey of plant-derived drugs in countries that host the WHO-Traditional medicinal Centres indicated that out of 122 compound identified 80\% were derived from 94 plant species. Some of the drugs obtained in this approach are: sodium cromoglycate, a bronchodilator from khellin (Ammi visnaga), Metformin, an antidiabetic from galegine (Galega officinalis), verapamil a antihypertensive from papaverine (Papaver somniferum), aspirin an analgesic from salicin (willow bark), atorvastatin from mevastatin (Penicillium citrinum), (Figure 5). Malaria remains one of the biggest challenges faced by the mankind, and there is a continuous search for an effective drug. The isolation of quinine from cinchona bark was reported in 1820 by the French pharmacists Caventou and Pelletier. Quinine formed the basis for the synthesis of the commonly used antimalarial drugs. Another antimalarial drug developed from plant lead is artemisinin obtained from Artemisia annua. There analogues are used in many countries for the treatment of malaria.

Other noteworthy drugs developed from traditional medicinal plants are: Reserpine an antihypertensive drug from Rauvolfia serpentina, Ephedrine from Ephedra sinica used as the basis for the synthesis of the anti-asthmatic drug salbutamol and salmeterol, tubocurarine a muscle 


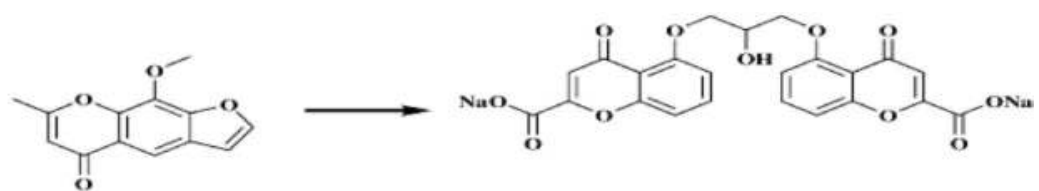

Khellin

Sodium Cromoglycate
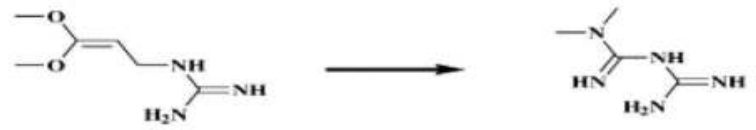

Galegine

Metformin
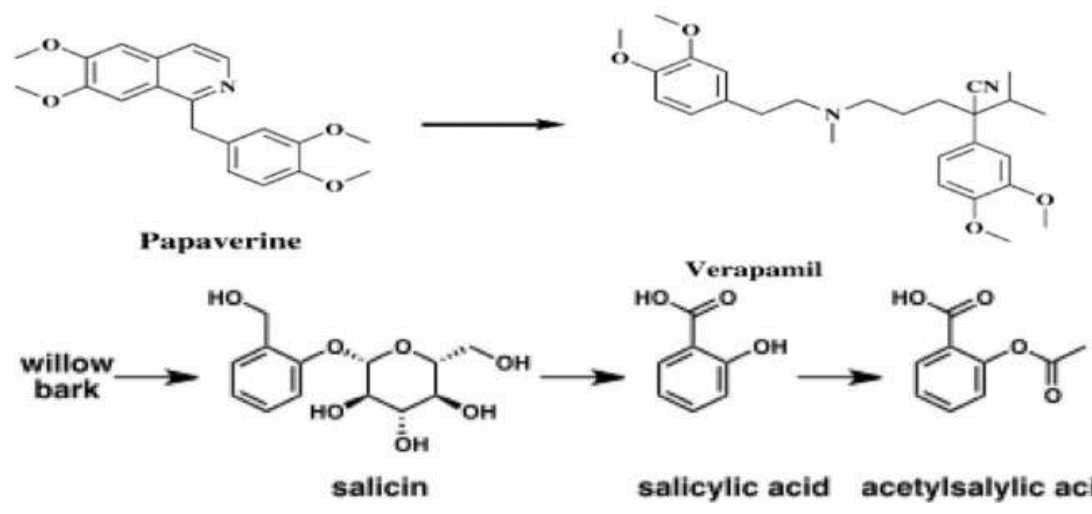

salicylic acid acetylsalylic acid

Figure 5. Drugs developed from plant lead molecule.

relaxant, from Curare species. A number of anticancer agents have been obtained from plants: vinblastine and vincristine from Catharanthus roseus and two clinically active compounds etoposide and teniposide, Paclitexel most exhilarating anticancer derived from Taxus species. (Figure 6).

In addition, recently, various other chemically active agents have gained attention and importantly placed in the arsenal of plant-derived anticancer agents. These are topotecan, irinotecan (CPT-11); belotecan and also their analogues 9-amino and 9-nitro camptochecin. These are semi-synthetic in nature, derived from camptochecin isolated from a Chinese ornamental tree camptotheca acuminate. One of the first plant-derived tubulin interactive compounds recently entered clinical trials, maytansine from the Ethiopian tree Maytenus serrata. This plant granted a new lease of life "warhead" (slightly modified), on a monoclonal antibody. The natural product chemists wondered if the compound was microbial in origin due to its similarity to the "ansa" antibiotic such as the rifamycins. Scientists at Takeda during 1977 reported very closely resembled the maytansinoids structure. Thereafter, compounds isolated from bacterium which was renamed as Actinosynnema pretiosum in fact similar to those isolated from other plant genera [8]. 


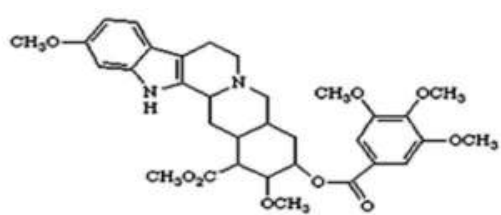

Reserpine

Rauwolfia serpentina

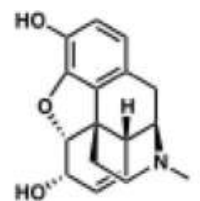

morphine

Papaver somniferous<smiles>COc1cc2c(c(OC)c1OC)-c1ccc(OC)c(=O)cc1[C@@H](NC(C)=O)CC2</smiles>

colchicine Colchicum autumnale

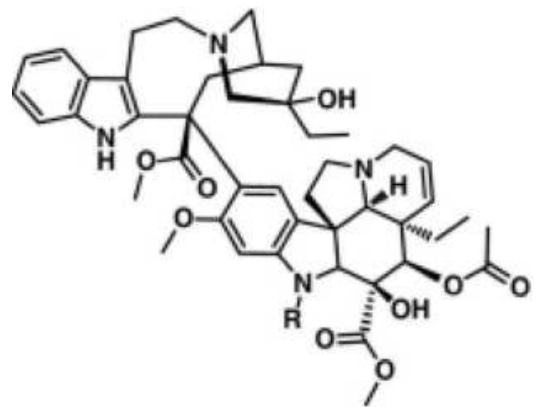

vincristine $\mathrm{R}=\mathrm{C}=\mathrm{O}$

vinblastine $\mathbf{R}=\mathbf{M e}$

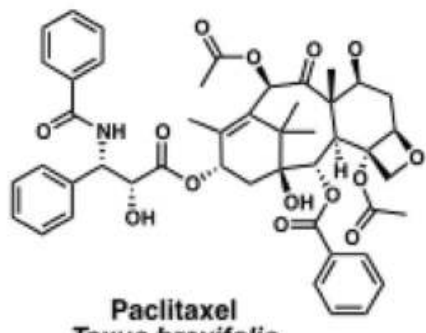

Taxus brevifolia

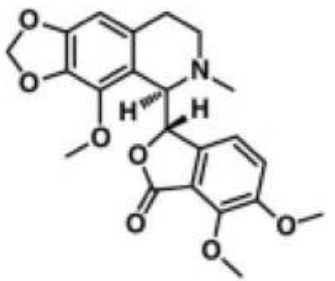

noscapine

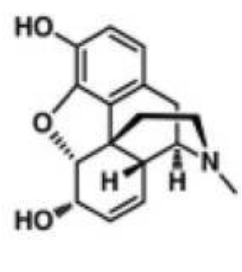

morphine

Figure 6. Plant-derived drugs.

\subsection{Animals}

Amphibians, reptiles and humans have been a fine source of drug. Epibatidine is a potent analgesic obtained from the skin of epipedobates tricolour (A frog). This drug is several times stronger than morphine. But the main snag is that the therapeutic dose of the drug is more close to its toxic dose that drives the development of synthetic analogue. Epibatidine has turned out to be important lead compound for potential novel painkillers. Teprotide isolated from the venom of the snake pit viper, Bothrops jararaca led to the design and synthesis of ACE inhibitor Captopril used in the management of hypertension. Another noteworthy finding 


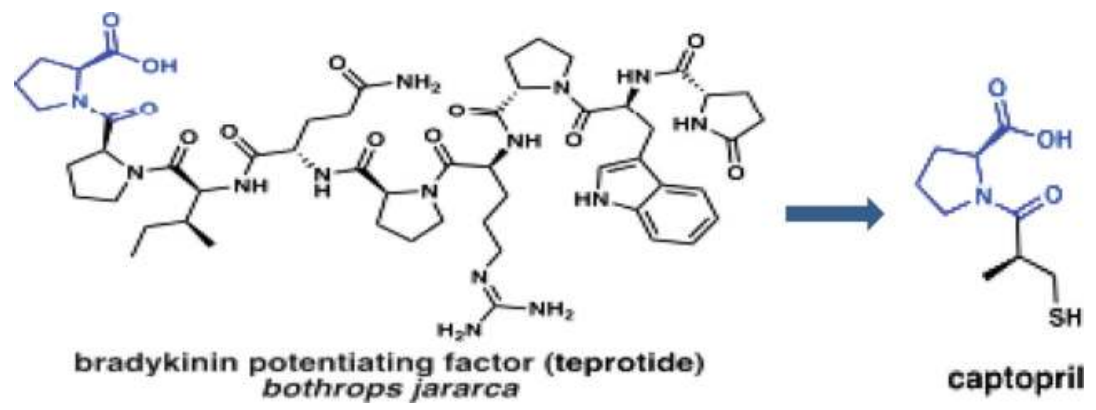

Figure 7. Drug from snake venom.

was the isolation of exendin-4 from the venom of Gila monster, Heloderma suspectum that leads to the development of Byetta an injectable antidiabetic drug to control type 2 diabetes. In 2009, a notable peptide having closeness to Human GLP-1 was approved for similar indication in Europe and then in Japan, in 2010, in the USA, under the name liraglutide [9] (Figure 7).

\subsection{Micro organisms}

The discovery of Penicillin from Penicillium notatum in 1929 by Fleming steers a new era in medicine "The Golden Era of Antibiotics" and accelerated the investigation of nature for bioactive agents. The microorganisms are the productive source of dissimilar bioactive metabolites and have turned out to be a vital source of drugs in Pharmaceutical industry. These are antibacterial agents: Penicillin from Penicillium species, cephalosporins from Cephalosporium acremonium, tetracycline, chloramphenicol, aminoglycosides, rafamycin, and so on. In addition to this immunosuppressive agents like cyclosporins from trichoderma, cholesterol lowering drug such as mevastatin (compactin; from Penicillium species) and lovastatin from Aspergillus species, anthelmintics and antiparasitic drugs like ivermectins from Streptomyces species are all originated from microorganisms [9].

Among the anticancer drugs, anticancer antibiotics plays a significant role in the chemotherapy. These are given in Table 1.

\subsection{Marine organisms}

Seventy five percent of the earth surface has been covered by water, but there is limited research as far as pharmacology of marine organisms is concerned. Many of these are still

\begin{tabular}{lll}
\hline S.No. & Anti-tumour antibiotic & Source \\
\hline 1 & Bleomycin & Streptomyces verticillus \\
2 & Mitomycin & Streptomyces caespitosus \\
3 & Daunomycin & Streptomyces peucetius \\
4 & Doxorubicin & Streptomyces peucetius var. caesius. \\
\hline
\end{tabular}

Table 1. Antitumor antibiotics. 
unexplored. Marine environment characterises various diverse resources towards new drugs to fight most major diseases like malaria and cancer. Marine environment also signifies an ecological resource consisting of a variety of aquatic plants and animals. Such aquatic organisms are screened for immunomodulatory, antibacterial, antifungal, antiinflammatory, antimicrobial, neuroprotective, anticancer, analgesic and antimalarial properties. These aquatic organisms are used for new drug developments mostly all over the world. Thus, under the marine pharmacology, there is further scope for research on the drugs of marine origin [10]. Marine pharmacology can be classified on the basis of source of the candidate drug:

- Genetically engineered marine organisms

- Manufacture of pharmaceuticals and nutraceuticals of marine origin

- Chemicals produced by or found in marine organisms shown to have a wide variety of applications as pharmaceuticals.

Classification of marine drugs on the basis of their action (Table 2).

Some of the drugs of marine origin approved for human use in different parts of the world are as follows (Table 3).

Given underneath are certain marine drugs that are now under Clinical Phase III trial (Table 4).

There are some marine drugs that are undergoing Phase II trial (Table 5).

Few drugs are also undergoing Clinical Phase I trial (Table 6).

\begin{tabular}{ll}
\hline Class & Marine drugs \\
\hline Antibacterial & $\begin{array}{l}\text { Eicosapentaenoic acid, isolated from Phaeodactylum tricornutum active against gram } \\
\text { positive and gram negative bacteria. }\end{array}$ \\
Anti-inflammatory & $\begin{array}{l}\text { The extracts and other parts of a Mediterranean sponge species Spongia officinalis showed } \\
\text { anti-inflammatory activity in vivo. }\end{array}$ \\
Neuroprotective & Extracts of South Indian green seaweed Ulva reticulate having neuroprotective action. \\
Antiparasitic & $\begin{array}{l}\text { Extracts of Sarcotragus sp. known as Tunisian sponge showed in-vitro anti-leishmanial } \\
\text { activity. }\end{array}$ \\
Antiviral agents & $\begin{array}{l}\text { Exo-polysaccharide extracted from the Celtodoryx girardae has Anti-herpes simplex virus-1 } \\
\text { (HSV) activity. }\end{array}$ \\
Anticancer & $\begin{array}{l}\text { Bryostatin, obtained from the Bryozoan, Bugula neritina, some forms from sponges and } \\
\text { tunicates have cytotoxicity. }\end{array}$ \\
Analgesic & $\begin{array}{l}\text { Ziconotide, first US FDA approved analgesic of marine origin. } \\
\text { The cephalosporins are well-known antimicrobial agents with a marine source of origin. }\end{array}$ \\
Antimicrobial & Isonitrile containing antimalarial molecules have been extracted from the Acanthella sp., \\
Antimalarial activity &
\end{tabular}

Table 2. Classification of marine drugs. 


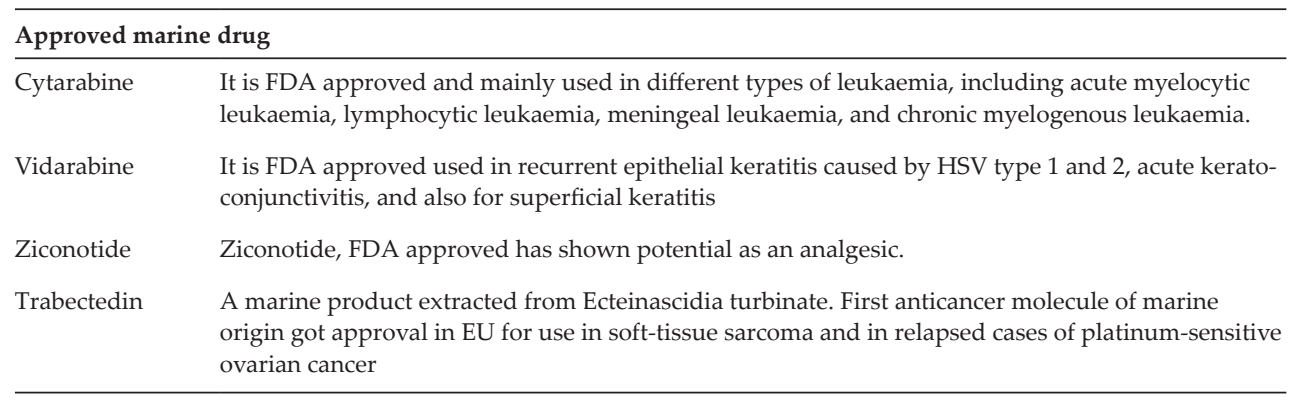

Table 3. Approved drugs of marine origin.

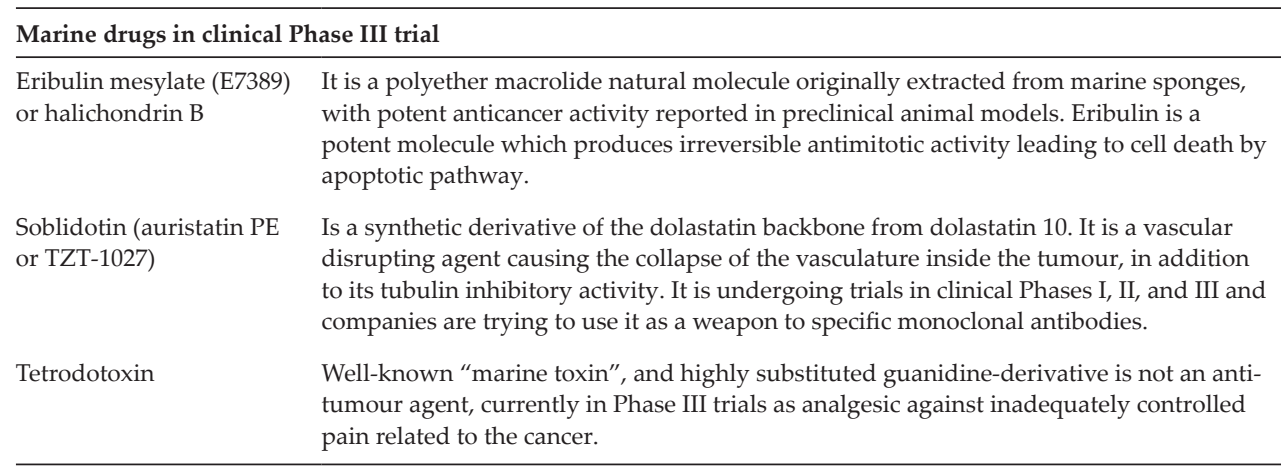

Table 4. Marine drugs in clinical Phase III trial.

\section{Marine drugs in clinical Phase II trial}

DMXBA (GTS-21)

[3-(2,4-dimethoxybenzylidene)

-anabaseine; GTS-21]

Plitidepsin

Elisidepsin (PM02734)

PM00104 (Zalypsis)

Plinabulin (NPI-2358)
It is a synthetic imitative of anabaseine, an alkaloid found in many species of aquatic worms of phylum nemertea. It is reported to be beneficial for improving cognition and sensory gating deficiency in a variety of laboratory animals.

It is a natural marine depsipeptide, currently obtained by total synthesis. It was primarily isolated from a tunicate Aplidium albicans found in the Mediterranean Sea. It is a highly potent apoptosis inducer.

It is a novel cyclic peptide derived from marine sources belonging to the Kahalalide family. It is now in Phase II with proof of antitumor potency with positive therapeutic index.

It is linked to jorumycin extracted from the Pacific nudibranch's (Jorunna funebris) skin and mucus as well as from renieramiycins extracted from varieties of sponges and tunicates. Preclinical in vivo studies indicated high antitumor activity in cells of breast, prostate and renal cancers with a modest antitumor action on colon cancer cells.

It is a fully laboratory made analog of the natural product halimide originally derived from marine Aspergillus sp. CNC-139 and phenylahistin extracted from Aspergillus ustus. It inhibits the polymerisation of tubulin, resulting in destabilisation of the vascular endothelial cells of the tumour. 
Marine drugs in clinical Phase II trial

ILX-651 (tasidotin or synthadotin)

Pseudopterosins
A synthetic derivative of dolastatin-15 and it inhibits assembly of tubulin. It is an orally active drug and has progressed to Phase II trials in different types of cancer.

A leading class of diterpene glycosides primarily extracted from the octocoral Pseudopterogorgia elisabethae. It is a strong phorbol myristate acetate inhibitor. In a double-blind, Phase II clinical trial, the drug was found to augment re-epithelialisation process in early wound repair process.

Table 5. Marine drugs in clinical Phase II trial.

\begin{tabular}{ll}
\hline Marine drugs in clinical Phase I trial \\
$\begin{array}{l}\text { Leconotide (AM-336, } \\
\begin{array}{l}\text {-conotoxin CVID) } \\
\text { Enfortumab vedotin }\end{array}\end{array}$ \\
$\begin{array}{l}\text { It is a peptide similar to Ziconotide and is undergoing Phase I trials for the treatment of } \\
\text { It is used in immunotherapy, and it is a combination of a fully human IgG1k antibody } \\
\text { and monomethyl auristatin E. }\end{array}$ \\
$\begin{array}{l}\text { Vorsetuzumab mafdotin } \\
\text { An antibody-drug conjugate, with monomethyl-auristatin F attached to the anti-CD70 } \\
\text { monoclonal humanised antibody 1F6. This molecule is presently being evaluated for its } \\
\text { value in relapsed and refractory non-Hodgkin's lymphoma in Phase I clinical trials and } \\
\text { also in metastatic renal cancer. }\end{array}$
\end{tabular}

Table 6. Marine drugs in clinical Phase I trial.

\section{Natural product drug discovery and development: an integrative approach}

An integrative approach comprising various discovery tools and novel discipline would definitely endow with an input in natural product drug discovery and development. Natural product can be envisaged to remain an indispensable component in the development of new drug. According to Lutz natural product not only complement synthetic molecule, they also exhibit drug-related features unsurpassable by any synthetic compound. An important attribute of natural product is their huge structure and chemical diversity. Another beneficial feature of natural product is their biological history. The natural products possess an inherent ability to interact with other molecules, which is a crucial precondition for making a drug. The natural product due to its sterically more complex structure exhibit advanced binding properly compared with synthetics. The natural products are perceived as "drug like-ness" and "biological friendliness" than totally synthetic molecule making them apposite lead candidates.

The process of drug discovery involves the identification of candidates, synthesis, screening, characterisation and assays for therapeutic efficacy, which in fact is a very lengthy and tedious process. Considering the success of natural products as source of new drugs, new technologies have emerged to facilitate the process. These technologies are combinatorial chemistry, high throughput screening (HTS), bioinformatics, proteomics and genomics. 
Other recently developed techniques are molecular diversity, compound library design, MMR based screening, QSAR and computer-aided drug design.

\subsection{Combinatorial chemistry}

Combinatorial chemistry involves the rapid synthesis or the computer simulation of a large number of different but often structurally related molecules or materials. In a combinatorial synthesis, the number of compounds made increases exponentially with the number of chemical steps. In a binary light-directed synthesis, $2 n$ compounds can be made in $\mathrm{n}$ chemical steps. Combinatorial chemistry is especially common in computer aided drug design (CADD) and can be done online with web-based software, such as mole inspiration.

\subsubsection{Principle of combinatorial chemistry}

Combinatorial chemistry is a technique by which large numbers of structurally distinct molecules may be synthesised in a time and submitted for pharmacological assay. The key of combinatorial chemistry is that a large range of analogues is synthesised using the same reaction conditions, the same reaction vessels. In this way, the chemist can synthesise many hundreds or thousands of compounds in one time instead of preparing only a few by simple methodology.

The conventional approach of synthesis is

$$
\mathbf{A}+\mathbf{B} \rightarrow \mathbf{A B}
$$

In contrast to this approach, combinatorial chemistry offer the potential to make every combination of compound $\mathrm{A} 1$ to An with compound B1 to Bn.

\section{Compound Libraries Screening Lead Compounds $\underset{\sim}{\longrightarrow}$ Optimization In -Vivo Studies}

The range of combinatorial techniques is highly diverse, and these products could be made individually in a parallel or in mixtures, using either solution or solid phase techniques.

Combinatorial Chemistry is used to synthesise large number of chemical compounds by combining sets of building blocks. Each newly synthesised compound's composition is slightly different from the previous one. A traditional chemist can synthesise 100-200 compounds per year. A combinatorial robotic system can produce in a year thousands or millions compounds, which can be tested for potential drug candidates in a high-throughput screening process [11].

\subsection{High throughput screening}

High throughput screening is a standard method for hit discovery for scientific experimentation in drug discovery and allied field. HTS uses robotics, data processing and control 
software, liquid handling devices and sensitive detectors making researchers to quickly conduct the biochemical, genetic or pharmacological tests. Employing HTS, it is comparatively trouble-free and swift to identify active compounds. HTS is hassle free technique that collects large amount of experimental data in a relatively short time [11].

\subsection{Bioinformatics, proteomics and genomics}

Genomics and proteomics in combination with combinatorial chemistry and high-throughput screening are helping to bring forward an unparalleled number of potential lead compounds. Proteomics includes technologies for protein mapping that is separating, distinguishing and quantifying the proteins in samples and also identification and characterisation of specific protein. The main protein mapping technology currently in use is two-dimensional polyacrylamide gel electrophoresis (2D-PAGE) that can resolve up to 2000 proteins in single gel.

Genomics is an area within genetics that concerns the sequencing and analysis of an organism's genome. The genome is the entire DNA content that is present within one cell of an organism. Experts in genomics strive to determine complete DNA sequences and perform genetic mapping to help understand disease. Since many diseases occurs due to failure of genes to perform correctly, genomics help to identify the genes involved in responsiveness to a given drug. Hence, genomics is an integral part of drug discovery [12].

\section{Challenges with natural products}

In spite of so many inherent advantages of these natural products for the synthesis of various molecules ranging from simple skeleton to highly complex chemical structures, they do have certain potential limitations.

- Drug discovery from natural products would eventually lead to its commercialisation. This may further burden the natural resource and consequently lead to undesirable environmental concerns. While synthesis of active molecule could be an option, not every molecule is amenable for complete synthesis. Hence, certain degree of dependence on lead resource would continue, for example, anticancer agent like etoposide, docetaxel, paclitaxel. It is expected that around 25,000 plant species would cease to exit by the end of this century.

- Another issue, the IPR protection related to the natural products is creating some confusion because the lead compounds are based on some linkage to traditional uses.

These processes impede the pace of discovery process at various levels. Challenges in the new drug developments are mainly due to:

i. Existing prototype for drug discovery in large pharmaceutical industries.

ii. Technical limitation of natural products 
According to Koehn and Carter, the unique feature/characters of the compound isolated from natural products are:

a. Increased steric complexity

b. Presence of large number chiral centres

c. Presence of greater number of oxygen atoms

d. Ratio of aromatic ring atoms to total heavy atoms, that is, low

e. Molecular rigidity is high

f. Wider distribution of molecular properties, such as molecular mass, $\mathrm{O} / \mathrm{w}$ partition coefficient and diversity of ring system [13].

It is presumed that large number of NP despite being biologically active and having favourable pharmacokinetic profile do not satisfy the criteria "drug likeness." The challenge is of building a physio-chemical tuned natural products library in line $\mathrm{c}$ the lead generation to promote natural products to their full potential. Therefore, ultimately, the biggest challenge is to find alternative drug ability criteria for the compound of natural origin, as they do not fit "rule of five" for to be drug like. As per rule of five propagated by Lipinski [14], a drug candidate should have:

i. Less than $10 \mathrm{H}$ bond acceptors

ii. Less than $5 \mathrm{H}$ bond acceptors

iii. Mol, wet $>500 \mathrm{Da}$

iv. PK of less than 5

\section{Conclusion}

Natural product drug discovery, ethno-pharmacology, traditional and attractive medicines are re-emerging as new strategic options. The chapter endeavoured that novel-natural products can be optimised on the basis of their biological activities using highly sophisticated combinatorial biosynthetic techniques, microbial genomes and screening process.

The chapter made efforts to provide short-lived imprint of the significance of natural products as bioactive molecules and also as pharmaceutical agents. On the advent of novel screening systems related to the discovery of genetic information accelerating the need to rapidly identify effective and novel lead structures as important necessity. It is certain that an important portion of these leads will remain to be resultant natural product.

As on today, comparative ease of access to plants now resulted in the discovery of a plantderived compounds, so far as the microbial sources are particularly important in the area of antibiotic. Further effort suggests, that marine organisms, and such group of organisms 
not much published, the marine-sourced fungi shall perform progressively significant role in the future. This role especially when given the impressive advances in the power of organic synthesis to report the supply problems intrinsic with this source material. With the arrival of genetic techniques that permit the isolation and expression of biosynthetic cases in the future, microbes and their marine invertebrate hosts might better be the new frontier towards natural products lead discovery. Plant endophytes also offer stimulating new resource.

Forthcoming features of antibiotic discovery and development include somewhat from a different perspective, a significant number of issues referred to in this chapter. Together with these novel sources to refurbished phenotypic screens that employ high-content imaging systems and that can run in microliter volumes, it might enable investigators to speedily evaluate the activity of individual agents and their potential.

\section{Author details}

Seema Kohli

Address all correspondence to: seemankohli@gmail.com

Pharmacy Department, K.N. Polytechnic College Jabalpur, Madhya Pradesh, India

\section{References}

[1] Lahlou M. The success of natural products in drug discovery. Pharmacology and Pharmacy. 2013;4:17-31

[2] Famsworth NR, Akerele O, Bingel AS, Soejarto DD, Guo Z. Medicinal plants in therapy. Bulletin of the World Health Organisation. 1985;63:965-981

[3] Ulrich-Merzenich G, Panek D, Zeiltor H, Velter H, Wagnes H. Drug development from natural products: Expoliting synergistic effects, Indian. The Journal of Experimental Biology. 2010;48:208-219

[4] Pawar HA. Natural product as a source of lead to the design of new drugs. Natural Products Chemistry \& Research. 2014;2(6):156. DOI: 10.4172/2329-6836.1000156

[5] Cragg GM, Newman DJ. Biodiversity: A continuing source of novel drug leads. Pure and Applied Chemistry. 2005;77(1):7-24

[6] Patwardhan B, Vaidya ADB. Natural products drug discovery: Accelerating the clinical candidate development using reverse pharmacology approaches, Indian. The Journal of Experimental Biology. 2010;48:220-227

[7] Bhutani KK, Gohil VSM. Natural products drug discovery research in India: Status and appraisal. Indian Journal of Experimental Biology. 2010;48:199-207 
[8] Balunas MJ, Douglas Kinghorn A. Drug discovery from medicinal plants. Life Sciences. 2005;78:431-441

[9] Cragg GM, Newman DJ. Natural products: A continue source of novel drug leads. Biochemica et Biophysica Acta. 2013;1830:3670-3695

[10] Malve H. Exploring the ocean for new drug developments marin pharmacology. Journal of Pharmacy \& Bioallied Sciences. 2016;8(2):83-91

[11] Rasheed A, Farhat R. Cominatorial Chemistry: A Review. International Journal of Pharmaceutical Sciences and Research. 2013;4(7):2502-2516

[12] Sharma N, Harikumar SL. Use of genomics and proteomics in pharmaceutical drug discovery and development. International Journal of Pharmacy and Pharmaceutical Sciences. 2013;3(15):975-1491

[13] Katiyar C. Drug discovery from plant sources: An integrated approach. An International Quarterly Journal of Research in Ayurveda. 2015;33(1):10-19

[14] Lipinski CA, Lombardo F, Dominy BW, Feeney PJ. Experimental and computational approaches to estimates solubility and permeability in drug discovery and development settings. Advanced Drug Delivery Reviews. 2001;46:3-26 